\title{
A Rare Case of Pyrethroid Poisoning Presenting as Auditory Hallucination
}

\author{
Ruhma Alia, d, Aditya Patel ${ }^{\mathrm{a}, \mathrm{d}}$, Modupeoluwa Owolabi ${ }^{\mathrm{a}}$, Rachel Bengart ${ }^{\mathrm{b}}$, \\ Emily Klein ${ }^{\mathrm{b}}$, Venkata Sugnanam ${ }^{\mathrm{c}}$
}

\begin{abstract}
Pyrethroid is an insecticide which is widely used for household pests. Even though they are relatively less toxic to humans, reports of accidental poisoning are not uncommon. Cases of anosmia, tremors and arrhythmias after pyrethroid exposure have been reported; however auditory hallucinations have not been presented yet. Herein, we present a case of auditory hallucination in the form of ringing in the ear after exposure to airborne pyrethrin in a 69-year-old male. The significance of this case report is related to the wide use of insecticides containing pyrethrin and highlights the need for physician awareness of this uncommon pathology.
\end{abstract}

Keywords: Insecticides; Pyrethroid poisoning; Auditory hallucinations

\section{Introduction}

Many common household insecticides are composed of pyrethroids. They appear as an alternative to the more toxic chemicals including organophosphates with less persistence and toxicity [1]. However, recent studies suggest that they are not entirely harmless and can lead to accidental or voluntary poisoning. Pyrethroid poisoning can occur through ingestion, inhalation, and absorption through the skin. Common side effects of pyrethroid poisoning include local erythema, itching, gastrointestinal manifestations, lacrimation, sweating and convulsions. Specific adverse effects including nephrotoxicity, hepatotoxicity, infertility, hyperglycemia, and hyperlipidemia have also been reported in the literature [2]. Below, we describe a rare presentation of acute auditory hallucinations induced by pyrethroid toxicity in

Manuscript submitted December 8, 2021, accepted January 26, 2022

Published online February 16, 2022

aDepartment of Internal Medicine, Saint Michael's Medical Center, Newark, NJ, USA

bUniversity of New England, ME, USA

'Department of Psychiatry, Saint Michael's Medical Center, Newark, NJ, USA ${ }^{\mathrm{d} C}$ Corresponding Author: Ruhma Ali and Aditya Patel, Department of Internal Medicine, Saint Michael's Medical Center, Newark, NJ 07102, USA. Email: raoruhma@gmail.com and adi3apr4@gmail.com

doi: https://doi.org/10.14740/jmc3878 a 69 -year-old male. To the best of our knowledge this is the first reported case of auditory hallucinations from pyrethroid exposure in the literature. Management was largely symptomatic, and the patient reported improvement in his symptoms after a few weeks of exposure-free period.

\section{Case Report}

\section{Investigations}

A 69-year-old male with a past medical history of coronary artery disease (CAD), myocardial infarction (MI) and interstitial lung disease, presented to the emergency department (ED) with complaints of hearing humming voices for the past few days. Patient reported living in a room which was infested with bed bugs and insects. The room was sprayed with pyrethrin containing insecticides to eliminate the bedbugs and insects 1 week ago. After the extermination the patient said that he went to sleep in the same room and after waking up he noticed itching, redness and swelling all over his skin with a constant, dull humming voice in both ears. Patient went to his primary care physician who gave him carbamide peroxide ear drops and diphenhydramine. The skin manifestations resolved but due to the persistent humming in the ear, he came to the ED for further workup. The patient denied any diarrhea, increased urination, lacrimation, change in vision, sweating, muscle weakness, tremors, numbness, tingling sensation, palpitations, confusion or any other cardiorespiratory, neurological, or gastrointestinal manifestations. The patient did not report any psychiatric history or any recent illicit drug use. He has never experienced similar symptoms before. On admission the vitals were stable. The temperature was $36.5^{\circ} \mathrm{C}$, pulse $91 /$ min, blood pressure 126/82 $\mathrm{mm} \mathrm{Hg}$ saturating $97 \%$ on room air. On physical examination the patient was awake, alert, and oriented to the person, place, and time. Conjunctiva were not injected; pupils were $4 \mathrm{~mm}$ bilaterally and reactive. Cranial nerves examination was grossly intact, the muscle strength was $5 / 5$ in all extremities, sensation to pinprick and vibration was normal. Gait was normal. Speech was clear and coherent. Glasgow coma scale (GCS) eye sub score was 4, GCS verbal sub score was 5 , and GCS motor sub score was 6 . Lungs were clear to auscultation; heart rate was regular without any murmurs and bowel sounds were normoactive. The remainder of the physical examination was unremarkable. 
Table 1. Laboratory Parameters

\begin{tabular}{lll}
\hline Laboratory parameters & Values & Reference range \\
\hline Sodium & 138 & $136-145 \mathrm{mmol} / \mathrm{L}$ \\
Potassium & 4.1 & $3.5-5.3 \mathrm{mmol} / \mathrm{L}$ \\
Chloride & 106 & $98-110 \mathrm{mmol} / \mathrm{L}$ \\
Blood urea nitrogen (BUN) & 15 & $6-24 \mathrm{mg} / \mathrm{dL}$ \\
Creatinine & 0.8 & $0.6-1.2 \mathrm{mg} / \mathrm{dL}$ \\
Aspartate transaminase (AST) & 15 & $10-36 \mathrm{U} / \mathrm{L}$ \\
Alanine transaminase (ALT) & 21 & $9-46 \mathrm{U} / \mathrm{L}$ \\
White blood cell (WBC) & 10.2 & $4.4-11 \times 10^{3} / \mu \mathrm{L}$ \\
Hemoglobin & 14.2 & $13.5-17.5 \mathrm{~g} / \mathrm{dL}$ \\
Platelets & 179 & $150-450 \times 10^{3} / \mu \mathrm{L}$ \\
Blood pH & 7.40 & $7.34-7.44$ \\
Cannabinoid & Negative & Negative \\
Cocaine & Negative & Negative \\
Phencyclidine & Negative & Negative \\
Thyroid stimulating hormone (TSH) & 0.894 & $0.400-4.500 \mu \mathrm{IU} / \mathrm{mL}$ \\
\hline Vitamin B12 & 458 & $180-914 \mathrm{pg} / \mathrm{mL}$ \\
\hline
\end{tabular}

\section{Diagnosis}

Initial labs did not show any electrolyte abnormality and are shown in Table 1. Urine drug screen was negative, and his ethanol level was $<3 \mathrm{mg} / \mathrm{dL}$. Urinalysis was normal. Computed tomography (CT) of the head was unremarkable. His electrocardiogram showed normal sinus rhythm. His thyroid profile was normal and vitamin b12 level was also within normal limits. For the consistent auditory disturbances and no identified focal neurologic cause, psychiatry was consulted. Recent exposure of pyrethrin and pyrethroid containing insecticide was the only trigger for auditory hallucinations presenting as persistent humming in the ears.

\section{Treatment}

Patient was started on risperidone $0.5 \mathrm{mg}$ twice daily. Patient was discharged with a follow-up visit advised after 2 weeks to assess the improvement in symptoms.

\section{Follow-up and outcomes}

At the follow-up visit 2 weeks after discharge from the hospital, the patient reported improvement in the auditory disturbances without any other symptoms.

\section{Discussion}

A pesticide is any substance used to repel, kill, or control unwanted pests. The term pesticides include insecticide for repelling insects, herbicide for unwanted vegetation and fungicide for the prevention of mold and mildews among others [3]. Insecticides are used in most homes and industries to control a variety of insects including bed bugs, mosquitoes, ants, fleas, and moths. Inappropriate use of insecticides can lead to adverse effects on the environment and humans [4]. The trend towards increased use of insecticides has led to higher chances of toxicity in exposed individuals [5]. Toxicity may result from oral ingestion, dermal route, inhalation, and injection. Pyrethroid is a compound synthesized from pyrethrins, which are a group of naturally occurring substances found in the flowers of Chrysanthemum cinerariaefolium [6]. They are potent, contact insecticides that cause rapid paralysis and death at a later stage in a variety of insects [7]. Certain limitations of natural pyrethrin extract include high rate of photodegradation and a rapid paralysis effect. This has led to the discovery of synthetic derivatives of pyrethroids without the limitations of parent compounds. While lethal to many insects including bed bugs, pyrethroids are considered safe in humans due to mammals' ability to rapidly metabolize and detoxify these agents [8]. As reported in this case however, they do have the potential to intoxicate humans and lead to a variety of symptoms ranging from mild local effects to life-threatening systemic symptoms. Pyrethroids exert their toxicity by acting on ion channels, particularly, sodium and chloride channels on nerve and muscle cells [9]. Pyrethroids act by delaying the closure of voltage-gated calcium channels therefore lowering the action potential threshold of these channels. This mechanism can result in repetitive firing of nerve and muscle cells. Pyrethroids also decrease current across chloride channels and at high levels can act as an inhibitor on gamma-aminobutyric acid (GABA)-gated chloride channels. This action, therefore, results in dysregulation of cellular excitability and 
synaptic inhibition, and can lead to a myriad of symptoms including the convulsant action of these insecticides. Animal studies of pyrethroid toxicity have also shown elevated levels of plasma epinephrine and norepinephrine in the exposed animals [10]. Reports of parenteral drug abuse associated with pyrethroid poisoning are sparse, however few cases have been illustrated in the literature of intravenous pyrethroid use making pyrethroids an under-reported substance of abuse if used intravenously [6].

Pyrethroid toxicity is generally divided into two syndromes, based on the type of pyrethroid involved in the exposure. Type I pyrethroids include allethrin, bifenthrin, permethrin, resmethrin and tetramethrin amongst others. Toxicity due to these compounds leads to what is known as the type I syndrome. Symptoms include a fine tremor, hyperexcitable reflexes, numbness of the tongue and dermal paresthesia if skin was exposed to the pyrethroid [2]. Type II pyrethroids include cyfluthrin, cypermethrin, deltamethrin and tralomethrin amongst others; and toxicity due to these compounds leads to a type II syndrome classically characterized by choreoathetosis, salivation, coarse tremor, and increased extensor tone [11]. The lipophilic nature of pyrethroids allows them to cross the blood brain barrier to gain access to the nervous system, and results in the various neurologic effects as described in type I and type II syndromes [8]. Ototoxic events following pyrethroid toxicity have been documented in the literature, however, auditory hallucinations from pyrethroid exposure have not been reported before.

The diagnosis of pyrethroid toxicity is largely based on clinical presentation coupled with exposure history. Signs and symptoms of pyrethroid toxicity may include gastrointestinal irritation, nausea, vomiting, diarrhea, dizziness, incoordination, syncope, headache, fatigue, and abnormal facial sensation. In more severe cases pulmonary edema, seizures, muscular paralysis, coma, and death due to respiratory paralysis can develop [12]. In our case the patient had exposure to the agent, developed symptoms shortly after exposure, had no psychiatric history and negative urine drug screen with normal laboratory values. After excluding all the possible differential diagnosis of auditory hallucination, the diagnosis of chemical-induced hallucination associated with pyrethroid exposure was made.

Treatment of pyrethroid toxicity depends upon symptomatic management. Short acting barbiturates can be used for convulsions. Gastric lavage and emetics can also be employed. As there is no specific antidote early diagnosis with supportive management is required [13]. Optimization of airway, breathing and circulation is crucial as with any acute poisoning preferably in a center that provides a higher level of circulatory and ventilatory support. Immediate decontamination of the skin has not proven to be beneficial but can be considered. The roles of drugs that target the effect of pyrethroid on chloride and sodium channels have been hypothesized, but larger prospective studies are required to formulate a guideline-directed medical therapy [14]. Poisoning due to pyrethroid may resemble organophosphate poisoning and can lead to misdiagnosis. It is essential to differentiate between the two chemicals to provide specific treatment. Pyrethroid does not inhibit plasma cholinesterase and treatment with atropine can lead to atropine toxicity in certain cases [15].

\section{Learning points}

In general, pyrethroids have been known to be relatively benign in humans however atypical presentations have been reported in the literature. To our knowledge this is the first reported case of pyrethrin poisoning presenting as auditory hallucinations. This case report highlights the importance of keeping pyrethrin toxicity in the differential for patients admitted with auditory hallucinations after exposure to this household chemical. The patients are usually managed symptomatically with favorable outcomes. Physicians should keep a high index of suspicion of pesticide poisoning in exposed individuals.

\section{Acknowledgments}

None to declare.

\section{Financial Disclosure}

None to declare.

\section{Conflict of Interest}

None to declare.

\section{Informed Consent}

The informed consent was obtained from the patient for the publication of the manuscript.

\section{Author Contributions}

Ruhma Ali MD, Aditya Patel MD, and Modupeoluwa Owolabi MD: diagnosis and management of the patient, discussion, writing and drafting of the case, and final approval of the case report. Rachel Bengart and Emily Klein: writing and drafting of the case, and final approval of the case report. Venkata Sugnanam MD: management of the patient, revision and final approval of the case report.

\section{Data Availability}

The authors declare that data supporting the findings of this study are available within the article.

\section{References}

1. Bhaskar EM, Moorthy S, Ganeshwala G, Abraham G. Cardiac conduction disturbance due to prallethrin (pyrethroid) poisoning. J Med Toxicol. 2010;6(1):27-30. 
2. Lucero B, Munoz-Quezada MT. Neurobehavioral, Neuromotor, and Neurocognitive Effects in Agricultural Workers and Their Children Exposed to Pyrethroid Pesticides: A Review. Front Hum Neurosci. 2021;15:648171.

3. Bronstein AC, Spyker DA, Cantilena LR, Jr., Rumack BH, Dart RC. 2011 Annual report of the American Association of Poison Control Centers' National Poison Data System (NPDS): 29th Annual Report. Clin Toxicol (Phila). 2012;50(10):911-1164.

4. Langley RL, Mort SA. Human exposures to pesticides in the United States. J Agromedicine. 2012;17(3):300315.

5. Badrane N, Askour M, Berechid K, Abidi K, Dendane T, Zeggwagh AA. Severe oral and intravenous insecticide mixture poisoning with diabetic ketoacidosis: a case report. BMC Res Notes. 2014;7:485.

6. Sharma P, Manning S, Baronia R, Mushtaq S. Pyrethroid as a substance of abuse. Case Rep Psychiatry. 2014;2014:169294.

7. Hodgson E. Toxins and venoms. Prog Mol Biol Transl Sci. 2012;112:373-415.

8. Panwar M, Usha G, Kumath M. Status epilepticus: An association with pyrethroid poisoning. Indian J Crit Care Med. 2013;17(2):119-120.
9. Kumar RV, Selvi K. Pyrethroid poisoning: an uncommon death. Journal of Forensic Medicine, Science and Law. 2014;23(1).

10. Cremer JE, Seville MP. Comparative effects of two pyrethroids, deltamethrin and cismethrin, on plasma catecholamines and on blood glucose and lactate. Toxicol Appl Pharmacol. 1982;66(1):124-133.

11. Akelma H, Kilic ET, Salik F, Bicak EA, Yektas A. Pyrethroid intoxication: a rare case report and literature review. Niger J Clin Pract. 2019;22(3):442-444.

12. Ramchandra AM, Chacko B, Victor PJ. Pyrethroid poisoning. Indian J Crit Care Med. 2019;23(Suppl 4):S267S271.

13. Gobba F, Abbacchini C. Anosmia after exposure to a pyrethrin-based insecticide: a case report. Int J Occup Med Environ Health. 2012;25(4):506-512.

14. Ray DE, Forshaw PJ. Pyrethroid insecticides: poisoning syndromes, synergies, and therapy. J Toxicol Clin Toxicol. 2000;38(2):95-101.

15. Yang PY, Lin JL, Hall AH, Tsao TC, Chern MS. Acute ingestion poisoning with insecticide formulations containing the pyrethroid permethrin, xylene, and surfactant: a review of 48 cases. J Toxicol Clin Toxicol. 2002; 40(2):107-113. 